\title{
Adnexal torsion in the third trimester of pregnancy: a case report and diagnostic value of MR imaging
}

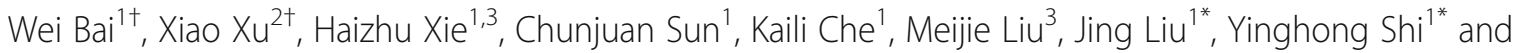
Heng $\mathrm{Ma}^{1,2,3^{*}}$

\begin{abstract}
Background: The torsion of normal adnexa is rare during pregnancy, especially in the third trimester. Nonspecific symptoms and signs as well as the limitations of ultrasound (US) make the diagnosis difficult, resulting in the loss of adnexa and fetal compromise. The magnetic resonance imaging (MRI) features of the torsion of normal adnexa are not classically described during pregnancy and only reported in a few cases. We find some different MRI features of the torsion of normal adnexa in late pregnancy and its diagnosis and treatment values are discussed in our report.

Case presentation: A 27-year-old woman at $31+5$ weeks' gestation presented to the emergency department with a three-day history of the left lower abdominal pain. US discovered a mass of $87 \times 61 \mathrm{~mm}$ in the left abdomen, but did not show whether the mass originated from the left ovary or the uterus. MRI showed the left ovary was increased in size to $82 \times 42 \times 85 \mathrm{~mm}$ with peripheral follicles. On fat-suppressed T2-weighted images, the signal intensity of the lesion was significantly decreased compared with the right ovary. The adjacent fallopian tube was found to be thickened. The radiologists diagnosed ovary infarction secondary to adnexal torsion. With the provisional diagnosis of adnexal torsion, the patient was taken to surgery. The left adnexal torsion was found during surgery. There was extensive hemorrhage and necrosis, so a left salpingo-oophorectomy was performed. The histopathology confirmed an extensively hemorrhagic fallopian tube and ovary with partial necrosis.
\end{abstract}

Conclusion: We believe MRI is helpful where US is indeterminate in diagnosis of the torsion of normal adnexa in advanced pregnancy. We found that aside from hyperintensity on fat-saturated T1-weighted images, the low signal intensity on T2-weighted images can also reflect adnexal hemorrhage in conjunction with the torsion of normal adnexa.

Keywords: Adnexal torsion, Magnetic resonance imaging, Pregnancy, Third trimester

\section{Background}

Adnexal torsion refers to the total or partial twist of the ovary around its vascular axis and can result in vascular compression and necrosis. A total of $13.7 \%$ of all adnexal torsion episodes are found in pregnant women [1]. Adnexal torsion occurs more frequently in the first and early second trimesters than in the third trimester [2]. The nonspecific clinical symptoms of adnexal torsion

\footnotetext{
*Correspondence: liujingyhd@126.com; shyhyt@163.com; hengma00@163.com

${ }^{+}$Wei Bai and Xiao Xu contributed equally to this work.

${ }^{1} Y$ uhuangding Hospital, Qingdao University School of Medicine, No. 20,

Yuhuangding East Road, Yantai 264000, Shandong Province, China

Full list of author information is available at the end of the article
}

and the anatomical and physiological alterations in advanced gestation complicate the diagnosis of adnexal torsion. Although ultrasound (US) is the accepted mainstay modality for adnexal torsion, it is extremely limited for visualization of the ovaries in women in their second and third trimesters of pregnancy [3]. These factors can delay the diagnosis of adnexal torsion and surgical management [4], resulting in the loss of adnexa and fetal compromise.

Magnetic resonance imaging (MRI) is a safe and effective method for making diagnosis in advanced gestation [3]. MRI can have a role where US is indeterminate. We present a case of the torsion of normal adnexa in

(c) The Author(s). 2020 Open Access This article is distributed under the terms of the Creative Commons Attribution 4.0 International License (http://creativecommons.org/licenses/by/4.0/), which permits unrestricted use, distribution, and reproduction in any medium, provided you give appropriate credit to the original author(s) and the source, provide a link to the Creative Commons license, and indicate if changes were made. The Creative Commons Public Domain Dedication waiver (http://creativecommons.org/publicdomain/zero/1.0/) applies to the data made available in this article, unless otherwise stated. 
advanced gestation. The MR imaging features are not classically described during pregnancy and only reported in a few cases. Compared with several previous case reports [5-7], some different MR imaging features of adnexal torsion in late pregnancy are discussed in our study as well as its diagnosis and treatment values.

\section{Case presentation}

A 27 -year-old woman at $31+5$ weeks' gestation (gravida 0 para1, G0P1) presented to the emergency department with a three-day history of the left lower abdominal pain. The pain was constant and getting progressively more severe. She did not complain of nausea or vomiting. There were no history of urinary symptoms, fevers, vaginal bleeding or uterine contraction. Ovarian hyperstimulation therapy was not performed. The patient's ovaries were noted to be normal on ultrasound prior to the start of pregnancy.

On examination, the patient's vital signs were stable. Abdominal palpation revealed a tender abdomen on the lower left side without signs of peritoneal irritation. The uterus was enlarged corresponding to $31+5$ weeks, and the cervix was closed with a little discharge. An obstetric US demonstrated the fetal parameters that corresponded to gestation with normal amniotic fluid and fetal activity. A heterogeneously hypoechoic mass of $87 \times 61 \mathrm{~mm}$ was discovered in the left abdomen adjacent to the left uterine border with unclear boundaries, which contains several non-echoic millimetric cysts. Doppler revealed a lack of perfusion within the mass (Fig. 1). US did not show whether the mass originated from the left ovary or the uterus.

To clarify the origin and the nature of the mass and decide the need for immediate surgical intervention, the patient subsequently underwent a prompt MRI scan. MRI showed the left ovary was increased in size to $82 \times$ $42 \times 85 \mathrm{~mm}$ with peripheral follicles. On fat-suppressed T2-weighted images, the signal intensity of the lesion was significantly decreased (Fig. 2) compared with the right ovary. On fat-saturated T1-weighted images, the enlarged left ovary had a signal intensity equal to that of muscle but was not homogeneous (Fig. 3). The adjacent fallopian tube was thickened, the diameter of the thickened tube was $11 \mathrm{~mm}$ (Fig. 2). Its signal intensity was similar to that of the left ovary. A small amount of fluid was detected between the mass and the uterus, as well as in the pouch of Douglas. The normal right ovary was also visualized. Combining the clinical symptoms with the US examination, three radiologists with about 20 years of experience in abdominal MR imaging diagnosed ovary infarction secondary to adnexal torsion.

With the provisional diagnosis of adnexal torsion, the patient was taken to surgery. During surgery, the left ovary was found to be twisted twice over $\left(720^{\circ}\right)$ around its pedicle and the left tube. The left ovary was $80 \times$ $60 \times 50 \mathrm{~mm}$ with a purple hue. The right adnexa and uterus were normal. As there was extensive hemorrhage and necrosis, a left salpingo-oophorectomy was performed. The histopathology confirmed an extensively hemorrhagic fallopian tube and ovary with partial necrosis (Fig. 4) and described multiple follicular cysts in left ovary. The pregnancy continued without problems, and the patient was discharged on postoperative day 3 .

\section{Discussion and conclusions}

Adnexal torsion is frequently associated with ovarian hyperstimulation therapy or ovarian masses. The torsion of normal adnexa is rare during pregnancy, especially in the third trimester. Early diagnosis and treatment are essential to save the adnexa and decrease maternal and fetal morbidity [8]. Adnexal torsion is difficult to diagnose during pregnancy, because its symptoms and signs are nonspecific and can be confusing when compared with other acute abdominal conditions. In the present case, we found that aside from hyperintensity on fatsaturated T1-weighted images, the low signal intensity

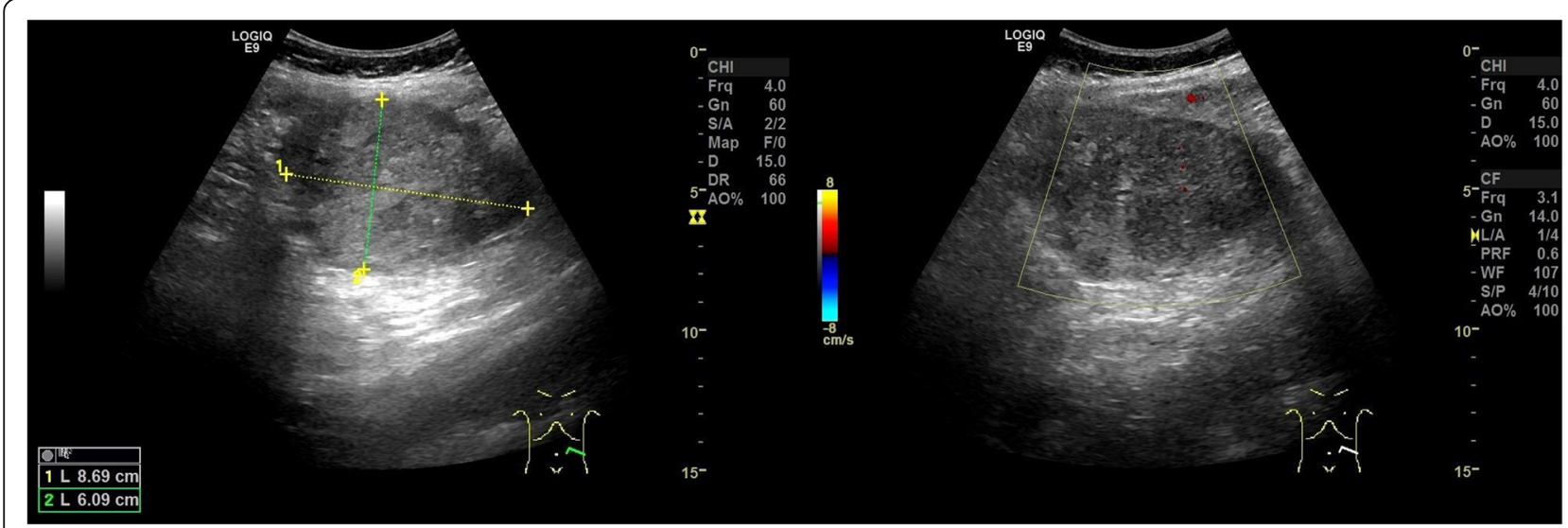

Fig. 1 Ultrasound showed a heterogeneously hypoechoic mass in the left abdomen and Doppler revealed a lack of perfusion within the mass 


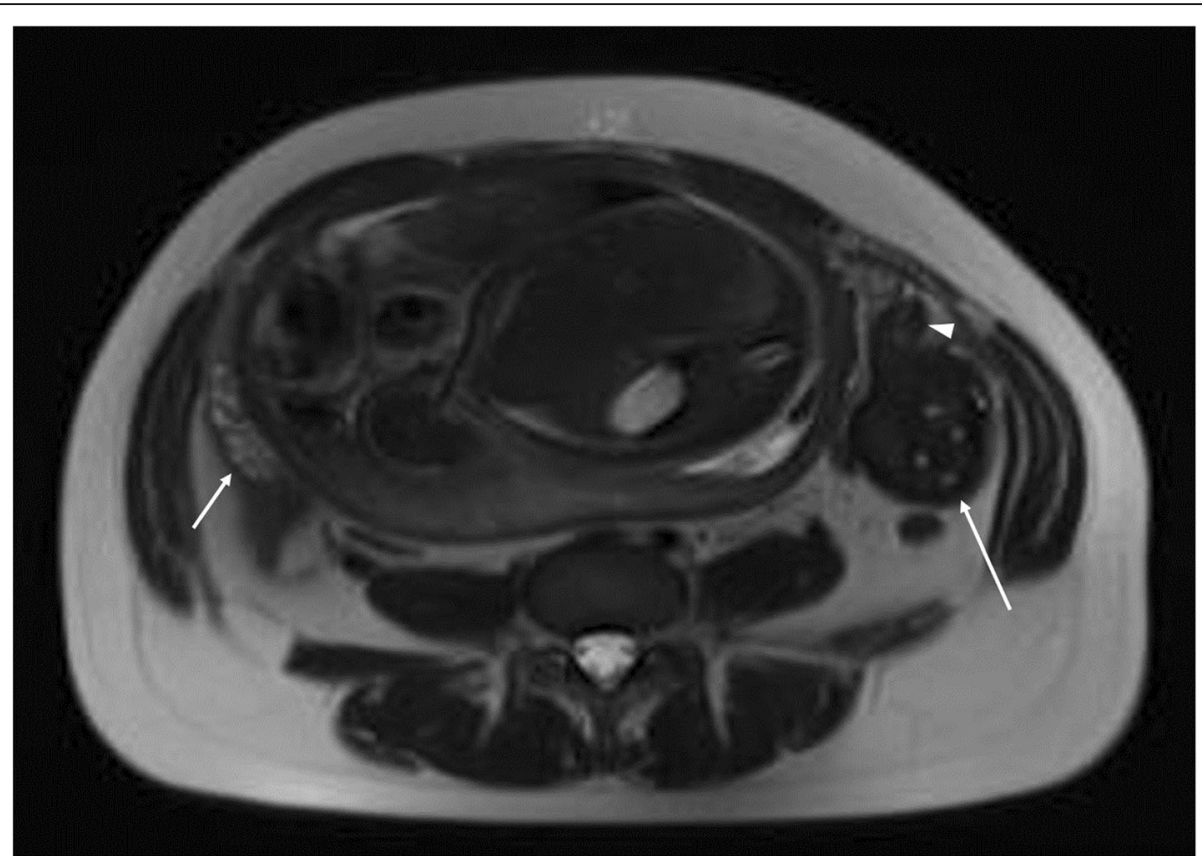

Fig. 2 Axial T2-weighted magnetic resonance imaging (MRI) shows an enlarged left ovary (long arrow) with multiple follicles and extensively low signal intensity compared with the normal right ovary (short arrow). The adjacent left fallopian tube was found to be thickened (arrowhead) with the diameter of $11 \mathrm{~mm}$

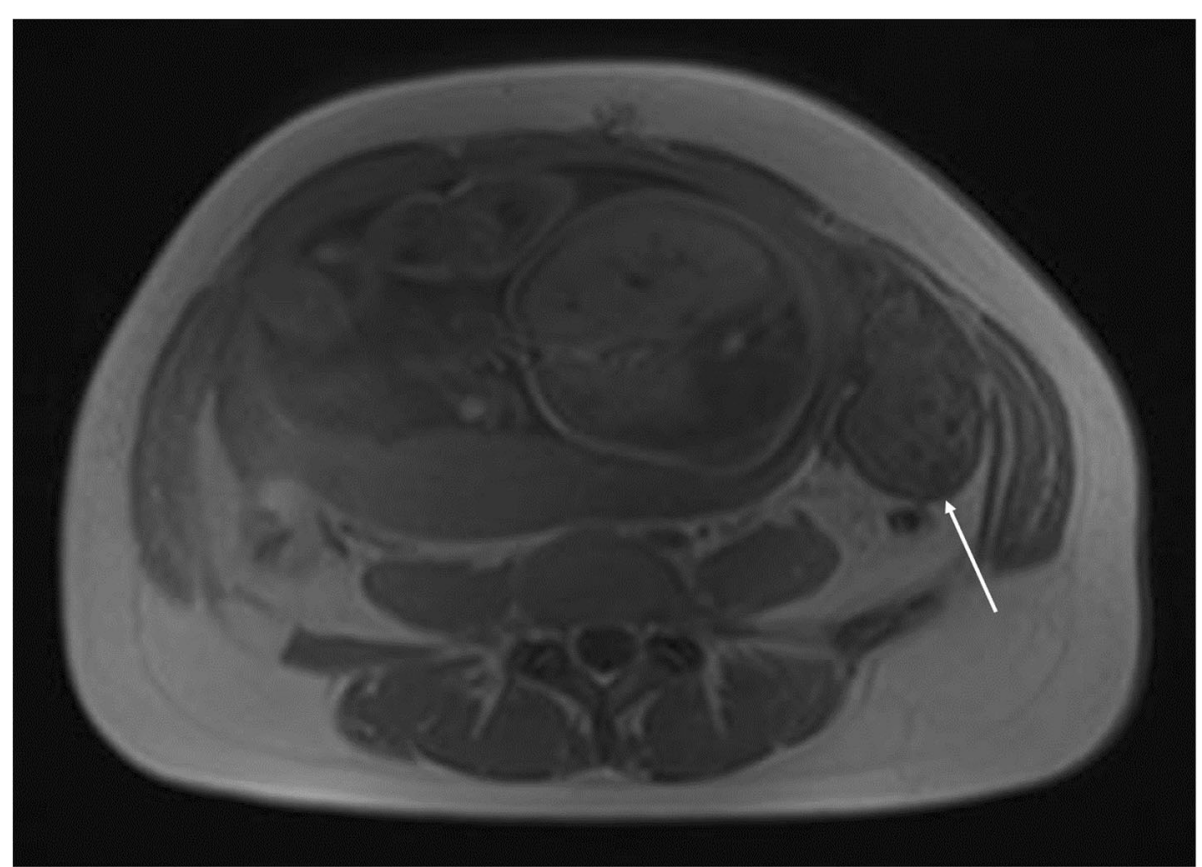

Fig. 3 Axial fat-saturated T1 weighted magnetic resonance imaging (MRI) shows the enlarged left ovary had a signal intensity equal to that of muscle but was not homogeneous (long arrow) 


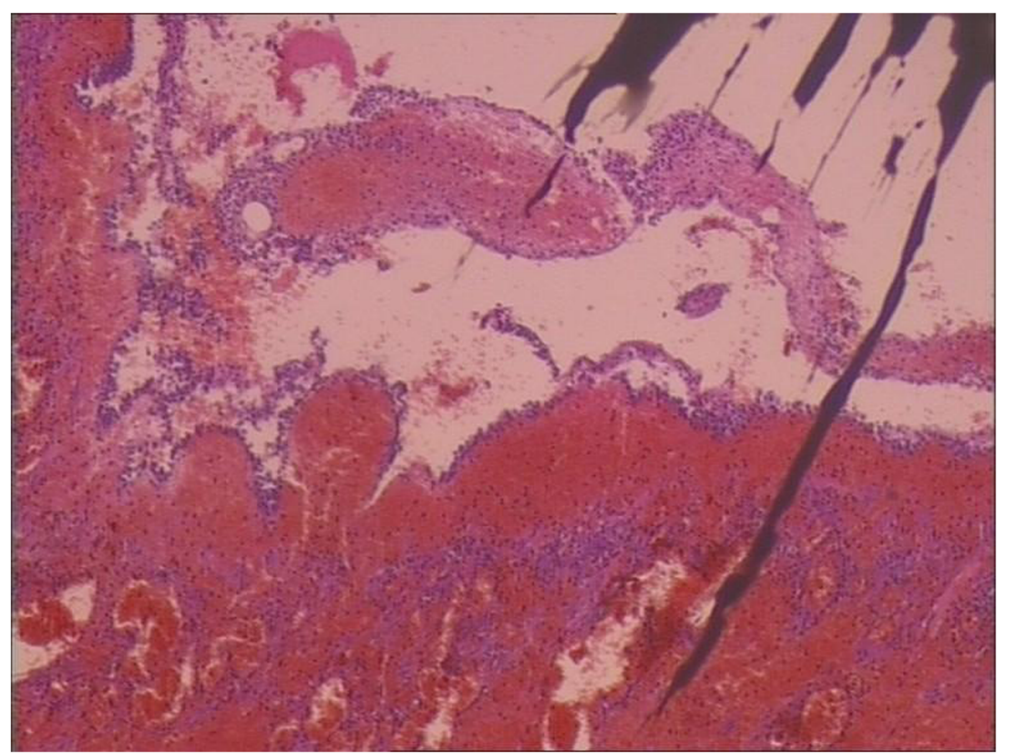

Fig. 4 Hemorrhage and necrosis of ovary H\&E $\times 20$. H\&E: hematoxylin and eosin

on T2-weighted images can also reflect adnexal hemorrhage when the torsion of normal adnexa occurred during pregnancy. This case demonstrates one instance where MRI is valuable in diagnosis of adnexal torsion in advanced gestation with equivocal sonographic findings.

MRI is a useful diagnostic modality when visualizing ovaries by routine US is difficult in the case of adnexal torsion suspected during the second and third trimesters of pregnancy [9]. The specific MRI imaging finding for adnexal torsion is tube thickening, which is related to congestion and edema with or without hemorrhagic infarction of the tube. The tube is considered thickened when the diameter of the tube exceeds $10 \mathrm{~mm} \mathrm{[10].}$ Béranger-Gibert et al. [11] reported that this sign was found in $90.0 \%$ of women with adnexal torsion. In our case, we found the thickened tube with the diameter of $11 \mathrm{~mm}$. Other useful findings include a small peritoneal effusion and the deviation of the uterus toward the involved side. However, these findings are not specific for adnexal torsion [10]. The MRI images of the adnexa can present varied characteristics in different phases because adnexal torsion is usually involved in a gradually ischemic process over time [12]. Initially, ovarian torsion causes mild ovarian congestion due to the occluded venous flow. MRI shows ovarian enlargement with diffusing stromal edema seen as hyperintensity on T2-weighted images. The ovary then undergoes hemorrhagic infarction, which is noted as hyperintensity on fat-saturated T1-weighted images, indicating nonviable ovary [13]. In our case, histopathology analysis described an extensively hemorrhagic fallopian tube and ovary with partial necrosis (Fig. 4). We did not detect hyperintensity on fat-saturated T1-weighted images in our case (Fig. 3), but extensively low signal intensity was observed on T2weighted images (Fig. 2). In several similar reports on the torsion of normal adnexa [5-7], the hemorrhagic and necrotic ovaries still appeared hyperintense on T2weighted images. Our finding differed from these reported cases. We speculate that the discrepancy may be related to different hemorrhage stages or bleeding amount. With the development of hemorrhagic necrosis, the hyperintensity of the stroma due to edema was invisible on T2-weighted images. When ovarian torsion with hemorrhagic necrosis occurred, immediate surgery was considered necessary.

US is a safe, accurate, fast, noninvasive, and inexpensive primary method for detecting adnexal torsion. The sonographic findings of adnexal torsion included the unilateral ovarian enlargement $(>4 \mathrm{~cm})$, uniform peripheral cystic structures, coexistent mass within the affected ovary, free pelvic fluid, lack of arterial or venous flow, and twisted vascular pedicle [14]. The presence of flow on color Doppler imaging dose not exclude torsion diagnosis. Pena et al. [15] reported that $60 \%$ of 21 surgically confirmed cases of ovarian torsion were normal on Doppler ultrasonography, leading to a delayed diagnosis up to 2 days. Factors such as increased bowel gas, small field of view (FOV), obesity and anatomical alterations in late pregnancy can hinder the proper visualization of adnexal regions, making the assessment of adnexal torsion difficult. In our case, the ovaries were displaced by the enlarged uterus in late pregnancy and no clear boundaries were found between the left uterine border and left ovary, so the operators were unable to determine whether the mass originated from the left ovary or the uterus and couldn't diagnose ovarian torsion definitively. 
Compared with US, MRI has different potential advantages, such as large FOV, multiplanar imaging, excellent soft tissue contrast, and ability to differentiate blood from other fluids. A study conducted by Béranger-Gibert et al. [11] reported that the accuracy of MRI was higher than $80 \%$ to diagnose the adnexal torsion in the context of acute pelvic pain present for less than $4 \mathrm{~h}$. Because it is difficult to obtain an emergent MRI on an unstable gravid patient with acute hemorrhage or pain, the use of MRI is limited in obstetric emergencies. However, the present MRI can be performed in a relatively short time without any specific patient preparation by using fast MR sequences which can decrease the time to approximately $30 \mathrm{~min}$. Maternal and fetal motion artifacts can be significantly reduced with acquisition time under $20 \mathrm{~s}$ during one maternal breath-hold [16]. According to our case, we believe that MRI is a useful problem-solving tool in diagnosis of adnexal torsion in advanced gestation with equivocal sonographic findings. Although MRI is more expensive than US, we still recommend that it should be used as a second examination in pregnant women with unclear diagnosis. To some extent, MRI can indicate the developmental stage of adnexal torsion, thereby providing guidance for clinical treatment.

In conclusion, the diagnosis of adnexal torsion in advanced gestation is difficult to make due to nonspecific symptoms and signs and the limitations of US. MRI is helpful where US is indeterminate in diagnosis of the torsion of normal adnexa in advanced pregnancy. MRI is sensitive in detecting adnexal hemorrhage when the torsion of the normal adnexa occurred during pregnancy, and on imaging is displayed as hyperintensity on fatsaturated T1-weighted images or low signal intensity on T2-weighted images.

\section{Abbreviations}

FOV: Small field of view; MRI: Magnetic resonance imaging; US: Ultrasound

\section{Acknowledgements}

The authors would like to acknowledge the staff of the Departments of Ultrasound and Radiology in Yuhuangding Hospital.

\section{Authors' contributions}

WB, XX, KLC and MLL were in charge of data acquisition, analysis and interpretation. WB was responsible for manuscript drafting. JL, YHS and HM Critically reviewed and revised the manuscript and supervised the study. HZX and CJS made a contribution to study conception and design. All authors contributed to the manuscript preparation, read and approved the final text.

\section{Funding}

This work was supported by National Natural Science Foundation of China [81671654 and 81571636]. This funding source had no role in in the design of the study, collection, analysis and interpretation of data, or in writing the manuscript.

\section{Availability of data and materials}

Not applicable.

Ethics approval and consent to participate Not applicable.

\section{Consent for publication}

Publication consent has been obtained from the patient in a written form.

\section{Competing interests}

The authors declare that they have no competing interests.

\section{Author details}

${ }^{1}$ Yuhuangding Hospital, Qingdao University School of Medicine, No. 20, Yuhuangding East Road, Yantai 264000, Shandong Province, China. ${ }^{2}$ Weifang Medical University, Weifang, Shandong Province, China. ${ }^{3}$ Binzhou Medical University, Yantai, Shandong Province, China.

Received: 4 October 2019 Accepted: 7 February 2020

Published online: 17 February 2020

References

1. Houry D, Abbott JT. Ovarian torsion: a fifteen-year review. Ann Emerg Med. 2001;38(2):156-9. https://doi.org/10.1067/mem.2001.114303.

2. Chang SD, Yen CF, Lo LM, Lee CL, Liang CC. Surgical intervention for maternal ovarian torsion in pregnancy. Taiwan J Obstet Gynecol. 2011;50(4): 458-62. https://doi.org/10.1016/j.tjog.2011.10.010.

3. Oto A, Ernst RD, Ghulmiyyah LM, Nishino TK, Hughes D, Chaljub G, Saade G. MR imaging in the triage of pregnant patients with acute abdominal and pelvic pain. Abdom Imaging. 2009;34(2):243-50. https://doi.org/10.1007/ s00261-008-9381-y.

4. Becker JH, de Graaf J, Vos CM. Torsion of the ovary: a known but frequently missed diagnosis. Eur J Emerg Med. 2009;16(3):124-6. https://doi.org/10. 1097/MEJ.0b013e32831cbaf8.

5. Born C, Wirth S, Stäbler A, Reiser M. Diagnosis of adnexal torsion in the third trimester of pregnancy: a case report. Abdom Imaging. 2004;29(1):123-7. https://doi.org/10.1007/s00261-003-0079-x.

6. Tayyar AT, Tayyar A, Atakul T, Şentürk MB, Cündübey CR, Tayyar M. Adnexal torsion in the third trimester of pregnancy: A challenging diagnosis. Int J Reprod Contracept Obstet Gynecol. 2017;6(7):3163-6. https://doi.org/10. 18203/2320-1770.jijrcog20172955.

7. Li C, Wang S, Tao X, Hu Y, Li X, Xiao X. Torsion of normal-sized ovary during late pregnancy: a case report and review of the literature. J Obstet Gynaecol Res. 2018;44(11):2110-4. https://doi.org/10.1111/jog.13758.

8. Silja A, Gowri V. Torsion of a normal ovary in the third trimester of pregnancy: a case report. J Med Case Rep. 2008;2:378. https://doi.org/10. 1186/1752-1947-2-378

9. Eskandar O, Eckford S, Watinson T. Safety of diagnostic imaging in pregnancy. Part 2: magnetic resonance imaging, ultrasound scanning and Doppler assessment. Obstet Gynecol. 2010;12(3):171-7. https://doi.org/10. 1576/toag.12.3.171.27599.

10. Rha SE, Byun JY, Jung SE, Jung Jl, Choi BG, Kim BS, Kim H, Lee JM. CT and MR imaging features of adnexal torsion. Radiographics. 2002;22(2):283-94. https://doi.org/10.1148/radiographics.22.2.g02mr02283.

11. Béranger-Gibert $S$, Sakly $H$, Ballester M, Rockall A, Bornes M, Bazot M, Daraï E, Thomassin-Naggara I. Diagnostic value of MR imaging in the diagnosis of adnexal torsion. Radiology. 2016;279(2):461-70. https://doi.org/10.1148/ radiol.2015150261.

12. Oelsner G, Shashar D. Adnexal torsion. Clin Obstet Gynecol. 2006;49(3):45963. https://doi.org/10.1097/00003081-200609000-00006.

13. Ghossain MA, Hachem K, Buy JN, Hourany-Rizk RG, Aoun NJ, HaddadZebouni S, Mansour F, Attieh E, Abboud J. Adnexal torsion: magnetic resonance findings in the viable adnexa with emphasis on stromal ovarian appearance. J Magn Reson Imaging. 2004;20(3):451-6. https://doi.org/10. 1002/jmri.20131.

14. Chang HC, Bhatt S, Dogra VS. Pearls and pitfalls in diagnosis of ovarian torsion. Radiographies. 2008;28(5):1355-68. https://doi.org/10.1148/rg. 285075130.

15. Pena JE, Ufberg D, Cooney N, Denis AL. Usefulness of Doppler so nography in the diagnosis of ovarian torsion. Fertil Steril. 2000;73(5):1047-50. https:// doi.org/10.1016/s0015-0282(00)00487-8.

16. Harrison BP, Crystal CS. Imaging modalities in obstetrics and gynecology. Emerg Med Clin North Am. 2003;21(3):711-35.

\section{Publisher's Note}

Springer Nature remains neutral with regard to jurisdictional claims in published maps and institutional affiliations. 